\title{
Auditoría de la información, punto de partida de la gestión del conocimiento
}

\author{
Por Susana Serrano González y Mònica Zapata Lluch
}

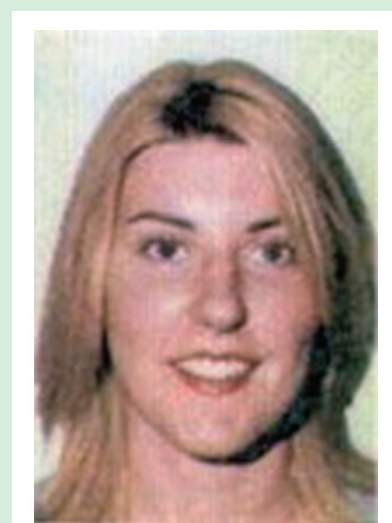

Susana Serrano González, diplomada en biblioteconomía y documentación, formación en gestión del conocimiento. Trabaja en GTD Ingeniería de Sistemas y de Software en el proyecto de gestión del conocimiento, responsable de la intranet y la web corporativa. Colabora en la Fundación Iberoamericana para la Gestión del Conocimiento como responsable de contenidos y de los "Encuentros con conocimiento en Barcelona".
Resumen: La auditoría de la información se posiciona como paso indispensable para llevar a cabo una correcta y eficaz gestión de la información y, en consecuencia, como requisito inexcusable para gestionar el conocimiento, recurso estratégico cada vez más importante de una organización.

Palabras clave: Auditoría de la información, Gestión de la información, Gestión del conocimiento.

\section{Title: The information audit: a starting point for knowledge management}

Abstract:

Information

auditing is becoming an essential step towards proper and efficient information management. As a consequence, it is a basic requisite for knowledge management, an increasingly important strategic resource for organisations.

Keywords: Information audit, Information management, Knowledge management.

Serrano González, Susana; Zapata Lluch, Mònica. "Auditoría de la información, punto de partida de la gestión del conocimiento”. En: El profesional de la información, 2003, julio-agosto, v. 12, n. 4, pp. 290-297.

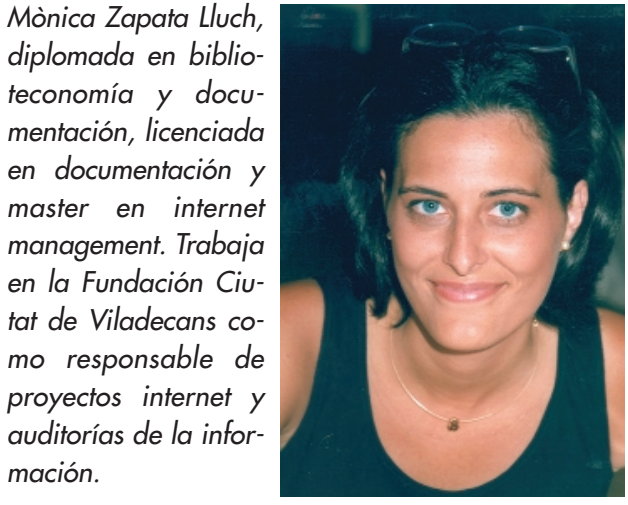

\section{Introducción}

Hablar de gestión del conocimiento no es nada nuevo; en los últimos años son muchas las empresas que han implantado programas con el único fin de conseguir este objetivo en su organización. Una vez más, han optado por las tecnologías de la información y las soluciones de software han vuelto a ser protagonistas. En poco tiempo ha podido valorarse como negativa esta opción, demostrándose que, para gestionar cualquier activo que posea una organización, hace falta algo más que tecnología, y que no todos los productos que se comercializan son válidos para todos los casos.

La mayoría de los programas se han basado en la implantación de intranets que han intentado facilitar la comunicación entre el personal, fomentar espacios de debate, documentar todos y cada uno de los procedimientos de la organización, poner a disposición de los empleados la política interna así como las estadísticas e indicadores de evaluación, etc. No entraremos en este momento en valoraciones sobre si esto es gestión del conocimiento o bien simplemente gestión de la información. Lo que parece evidente es que el conocimiento en sí mismo difícilmente puede gestionarse y que, en cambio, la información de la que disponemos sobre éste si puede ser gestionada.

En este contexto, la información toma un papel relevante como base de toda estrategia de gestión del conocimiento, pues la organización dispone de información sobre los conocimientos de sus empleados y debe aprender a gestionarla antes de plantearse la implantación de cualquier solución de gestión del conocimiento.

La correcta gestión de la información, entendida genéricamente como las actividades orientadas a controlar, almacenar y recuperar la información que posee 
"It has revolutionised the way I find technical information. When I have a question, it searches hundreds of IT titles to quickly give me the correct, up-to-date answer."

\section{Search the best IT books on line}

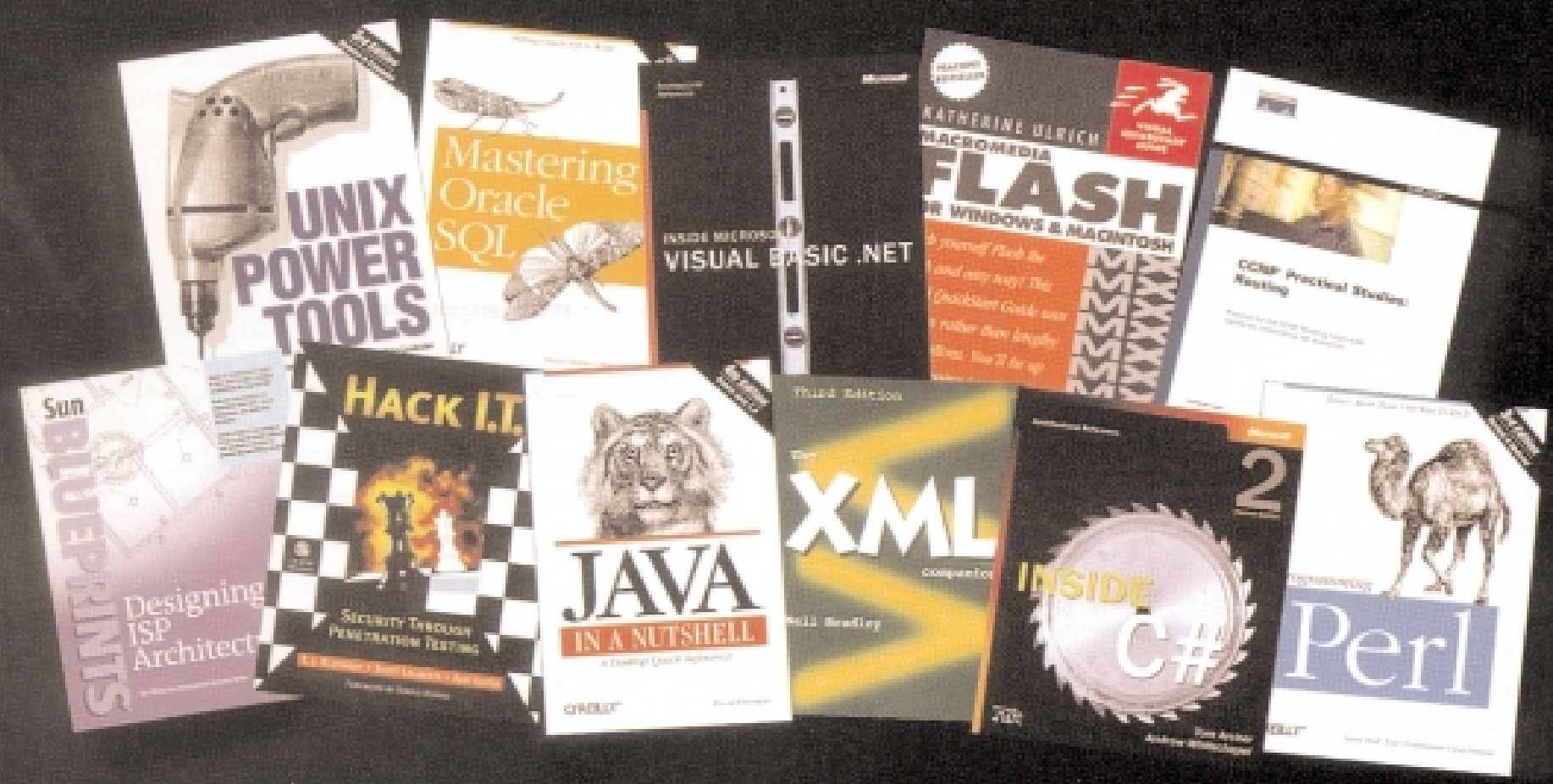

\section{IT answers in an instant}

Introducing Safari"m Tech Books Online"m, now available from ProQuest ${ }^{*}$. It is a fully-searchable database comprising the very best Information Technology content on the planet.
Hundreds of titles from the premier IT publishers are available on subjects ranging from XML to Networking to Java. It is cross-searchable, up-to-date and completely customisable.

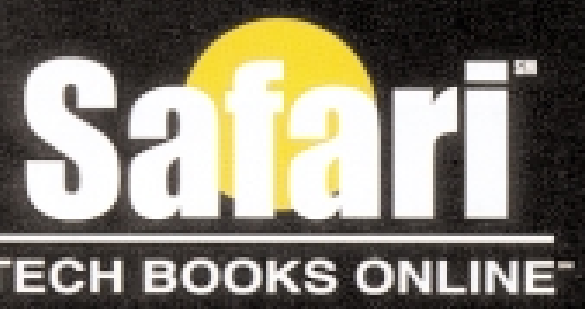

For more information visit: www.proquest.co.uk 
una organización se presenta, en consecuencia, como un elemento indispensable. Pero, ¿saben las organizaciones de qué información disponen?, ¿qué valor le otorgan a la información?, ¿quién es responsable de su gestión?, ¿qué relación hay entre el conocimiento, la información y el personal de una organización?, ¿es la tecnología un medio para gestionarlos?

\section{Tecnología vs. información}

En las últimas décadas las empresas han incorporado nuevas tecnologías de la información como componente eje de sus sistemas de información, hasta el punto de acabar equiparándolo con el sistema o red informática de la empresa. Pese a que su implantación ha sido progresiva, en contadas ocasiones ha sido llevada a cabo de forma correcta, ya que la confusión existente entre información e informática ha conllevado muchos errores.

La importancia que se le ha otorgado a la tecnología la ha acabado convirtiendo en el objetivo a alcanzar por la organización y con este razonamiento se ha llegado al grave convencimiento de que la tecnología de forma aislada puede mejorar el funcionamiento de ésta, el control de sus procesos y la gestión de sus recursos. De este modo, la empresa mejor dotada tecnológicamente debería ser la que alcanzara resultados más óptimos, pues su gestión debería ser mejor. La tecnología se presenta por lo tanto como un evidente factor de éxito.

Sin embargo, la mayoría de organizaciones que han adoptado esta dinámica han podido comprobar que no todos los objetivos establecidos estaban en manos de la tecnología y, en el peor de los casos, que el sistema de información diseñado no lograba alinearse ni con la cultura de la organización ni con su política. En este sentido, los fracasos de los sistemas de información se han sucedido en los últimos años, demostrando que la tecnología por sí sola sirve de poco, pues ésta nunca debe ser la finalidad en sí misma, sino el

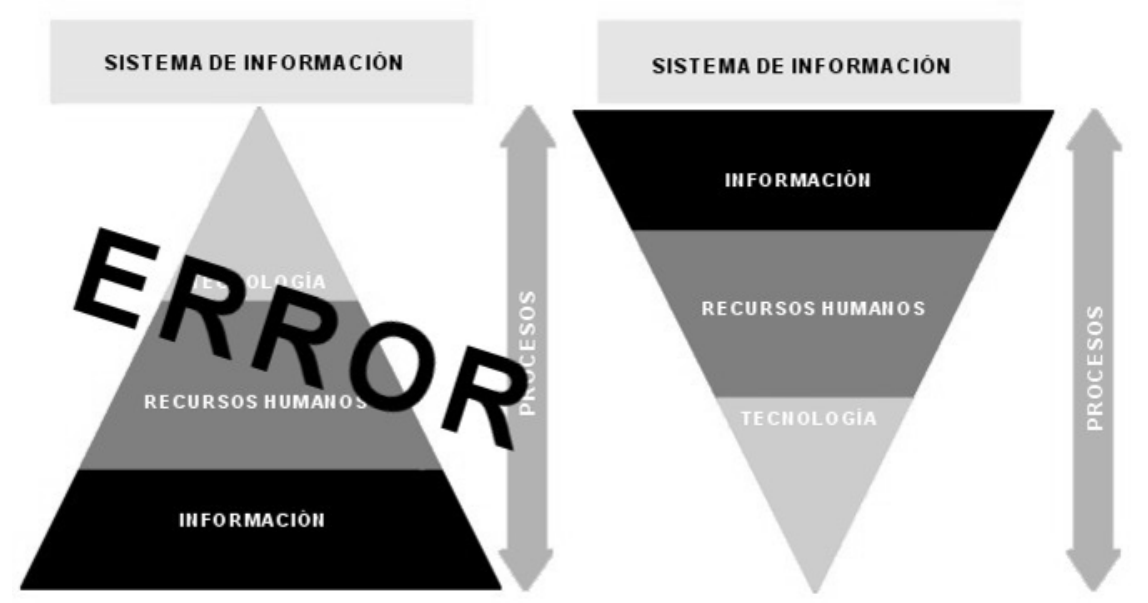

Figura 1 medio para que un sistema de información cumpla el propósito para el que ha sido creado y optimice los resultados previstos. Contrariamente al paradigma que durante años ha ido tomando fuerza, la tecnología individualmente es incapaz de agregar valor.

Otro de los errores ha sido confiar en que una misma tecnología puede funcionar en organizaciones distintas, independientemente de su estrategia de negocio, del personal que las compone o de la información que gestionan. De esta forma, las aplicaciones se han comercializado de forma masiva independientemente del lugar en que fueran a utilizarse y de las funciones que quisieran asignárseles. Las malas experiencias se han sucedido y las pérdidas económicas que ello ha supuesto son alarmantes.

\section{«Contrariamente al paradigma que durante años ha ido to- mando fuerza, la tecnología in- dividualmente es incapaz de agregar valor»}

El desacierto que se produce pasa por desarrollar el sistema de información independientemente de la cultura organizativa. Una organización consta de una estructura, una cultura, una política, una comunicación y unas relaciones tanto internas como externas que la diferencian del resto. Es decir, es una entidad tan sumamente única que aplicar una solución genérica puede resultar un absoluto fracaso. Sólo cuando se desarrolla un sistema de información adecuado a la organización a la que ha de servir puede ser considerado útil.

Aún así, no se han dejado de impulsar departamentos que bajo distintos nombres se han preocupado únicamente de implantar el mejor hardware y los más reconocidos estándares de software, así como de mejorar las redes y comunicaciones internas. En la mayoría de ocasiones estos departamentos, dirigidos casi de forma exclusiva por perfiles tecnológicos, han obviado el resto de componentes que deberían conformar el sistema de información de cualquier organización y en consecuencia han agravado aún más el problema de base.

Además, pese a que se alardee de implantar extraordinarios sistemas de información e invertir ingentes cantidades de dinero para desarrollarlos, la información raramente se considera en su diseño y planificación. Esto nos lleva a considerar dónde radica el problema: la finali- 
dad del sistema de información raras veces es la mejora de la gestión de la información. Confundir información con datos, pensar que es fácilmente almacenable y, en definitiva, considerar que la tecnología es la única vía para mejorar su gestión son algunos de las disfunciones detectadas.

La solución pasa pues por invertir la estrategia de desarrollo de sistemas de información que hasta el momento se ha venido llevando a cabo (figura 1).

\section{Información y organización}

Es evidente que la información, a pesar de ser un recurso vital para la organización, no está lo suficientemente valorada por la estrategia empresarial. Es un actor imprescindible en la generación de negocio y en la transformación del mismo, ya que cambiar la situación de negocio de una organización pasa siempre por modificar también su entorno informativo. La visión tradicional de la información como un medio de control y de coordinación interna en la empresa debe avanzar para pasar a ser vista como una fuente de generación de valor para el negocio, añadiendo competitividad a los productos y logrando unos procesos más eficaces.

En una organización, la mejora de plazos, costes y calidad está presente en la cadena de valor a través del tratamiento eficiente de la información, ya que la disponibilidad de determinada información influye de forma directa en el modo en que se desarrolla el trabajo dentro de ésta.

Por otro lado, el valor añadido de los procesos de interacción entre las personas que intercambian información en el entorno empresarial es el factor que determina el nivel de eficiencia en la utilización de la información en la organización. De hecho, la comunicación interna (ascendente, descendente y horizontal) asegura la identificación de mejoras, ahorro de costes $\mathrm{y}$, como consecuencia, es una fuente de generación de oportunidades para la organización.

Así pues, la aplicación de una estrategia empresarial teniendo en cuenta la información que fluye en el entorno de la organización, sirve no sólo como medio de detección de procesos que se dan en la empresa, sino también como vía para su optimización y reducción de riesgos, por lo que parece claro que los cambios organizacionales pasan inevitablemente por tener la información en cuenta. Sin embargo, no toda la información que discurre por una organización contribuye a la mejora de los elementos descritos. Para ello es importante determinar el valor de la información y en consecuencia analizar su pertinencia, su precisión o grado de focalización, su oportunidad, su singularidad, su puntualidad, su fiabilidad y evidentemente su apli- cabilidad. Saber desechar aquella información no estratégica para la organización será, pues, otra de las claves de éxito.

\section{Gestión del conocimiento vs. gestión de la información}

El conocimiento entra en juego en una organización cuando las personas aplican su saber y lo conjugan con la información de que disponen para desarrollar su trabajo. Por eso es necesario que las organizaciones busquen esa conexión perfecta entre el conocimiento que poseen y los problemas a los que se enfrentan día a día, ya que si esto no ocurre, no conseguirán explotar el conocimiento y por lo tanto, éste no será productivo.

Es conveniente que el primer paso a realizar antes de desarrollar cualquier estrategia de gestión del conocimiento sea identificar aquél existente en la organización y dónde es necesario para apoyar sus decisiones y acciones. Sólo de este modo la organización será capaz de valorar la importancia del conocimiento del que dispone como recurso.

El valor del conocimiento no es nuevo, aunque actualmente está considerado como el activo más importante a gestionar y a controlar pero, realmente, ¿se está haciendo referencia al conocimiento o a la información? Esta dualidad ha sido planteada en incontables ocasiones y cabe hacer una vez más una distinción entre los dos tipos de conocimiento para entenderla:

-Tácito: resultado de la experiencia, la creatividad, la intuición; es intrínseco a cada persona.

-Explícito: saber que puede reflejarse, transmitirse o compartirse entre las personas sin ninguna dificultad; son conocimientos técnicos que tienen capacidad de ser transmitidos a la organización.

Esto nos lleva a pensar que, con toda seguridad, gran parte del conocimiento explicito que comparta el personal de una organización acabará convirtiéndose en información que fluya a través de ella y que indudablemente en estos casos estaremos refiriéndonos a la necesidad de una estrategia de gestión de la información.

Por otro lado, y partiendo de la idea de que el conocimiento tácito como tal no puede ser gestionado, desarrollar una estrategia de gestión del conocimiento que considere la gestión tanto del tácito como del explícito será un error. Se debe centrar por lo tanto en la gestión del conocimiento explícito y en este sentido debe abogar por:

- Mediar para que pueda ser formalizado y, en consecuencia, reutilizado tantas veces como sea necesario independientemente de quién lo haya generado. 
-Fomentar la transmisión entre los miembros de una organización mediante la comunicación.

En relación a este último punto, cabe destacar que en ocasiones el exceso de información existente puede dificultar la comunicación y, en definitiva, la transmisión del conocimiento. En este sentido, la solución radica en intentar dotar de contexto, orientar y dar sentido a la comunicación para que las organizaciones aprovechen sólo aquella información que les es útil, ya que únicamente de éste modo se logrará su uso inteligente. Además, existen tantos factores que pueden hacer que la información se deteriore y llegue a descontextualizarse que, para evitar que esto ocurra, uno de los revulsivos será una buena política de gestión de la información.

La estrategia de gestión del conocimiento tiene, por tanto, como pilares los recursos humanos (en calidad de generadores, poseedores y transmisores de conocimiento), los procesos y la tecnología entendida como medio, pero no puede olvidarse que la gestión de la información es la base que sustenta esta estrategia y la clave del éxito de un proyecto de gestión del conocimiento.

Pese a la evidencia de que la información es un recurso elemental dentro de una organización, raramente se tiene conciencia de que como tal debe ser gestionada y evaluada del mismo modo que cualquier otro. Esto a menudo ha ocasionado que su gestión quedara reducida a almacenar y difundir los recursos informativos, no teniendo en cuenta que también supone:

— ser consciente de para quién se está gestionando,

- conocer qué necesidades tiene cada uno de los miembros de la organización para el desarrollo de sus funciones,

- analizar con qué información cuenta y el uso que hace de ella, $y$

- detectar posibles vacíos o carencias.

Pero además, una gestión de la información eficaz facilitará no sólo la consecución de estos objetivos o la alineación con la estrategia de negocio de la organización, sino también la generación de conocimiento, pues sólo si su personal dispone de información puntual (just in time) y de calidad podrá seguir generando conocimiento útil para la organización.

El valor diferencial en las organizaciones consistirá cada vez más en dotar el conocimiento con una información de calidad. Para ello será necesario realizar un análisis de la información que se posee. Sólo si se realiza una selección cualitativa se obtendrá un conocimiento de calidad. Sin embargo, no será posible desarrollar una buena estrategia de gestión de la infor-

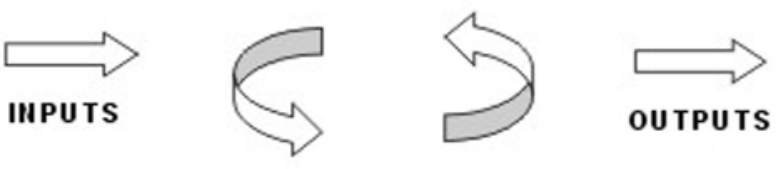

Figura 2

mación sin analizar previamente aquella que tiene la organización o, lo que es lo mismo, auditarla.

\section{Auditoría de la información}

Es un proceso que permite detectar, controlar y evaluar la información que existe en una organización, así como los flujos de información que en ésta discurren, el uso que se hace de ella y su adecuación a las necesidades de su personal y a los objetivos de la organización. De esta forma resolveremos en primer lugar la incertidumbre sobre qué información tiene la organización y dónde está situada, lo cual nos ayudará a identificar:

-Duplicidades. Entre unidades de una misma organización muchas veces se crea, mantiene y almacena la misma información de forma independiente.

- Carencias. Del mismo modo que en el caso anterior, en ocasiones, no compartir la información hace que detectemos vacíos que pueden perjudicar el correcto funcionamiento de determinadas unidades de negocio dentro de la entidad.

-Inconsistencias. Mantener la misma información de modo independiente puede dar lugar a informaciones dispares o contradictorias.

En segundo término, la auditoría nos permitirá diagnosticar qué uso se hace de la información y en definitiva la importancia que se le otorga. Además, esto nos dará la posibilidad de saber quién la está usando en cada caso y, lo que es más importante, para qué, con lo que podremos detectar aquellos puntos críticos de la cadena de valor donde el uso de información es esencial. Con la auditoría podremos profundizar en definitiva en la organización, la estructura y la distribución de la información, y lo que es más importante, en cómo se procesa en cada unidad.

Paralelamente, nos permitirá detectar los inputs o información que procede del exterior, cómo discurre por la organización y su relación con aquella generada internamente y, finalmente, los outputs o información proyectada hacia el entorno y en qué casos este flujo genera retorno (figura 2). 


\section{En primera línea en Sistemas de Información y Gestión del Conocimiento.}

[ Informática Documental

Internet, Intranet

Edición de Bases de Datos en CD-Rom

- Sistema Integrado de Gestión Bibliotecaria Absys

- Catalogación Retrospectiva

- Sistema Integrado de Gestión de Centros Archivísticos Albalá 
Por otra parte, nos dará la posibilidad de considerar las necesidades concretas de información de los miembros de la organización, así como las de sus proveedores y las de sus clientes. En este sentido, siempre es recomendable desarrollar reuniones colectivas e individuales con los distintos niveles de la organización, con el objetivo de detectar las necesidades que no quedan cubiertas, así como los requisitos que debería cumplir la información y no cubre.

El análisis del comportamiento informacional del personal servirá para reflejar la cultura de la organización, y su radiografía nos ayudará, en último término, a detectar las áreas de mejora. De este modo, otra ventaja de desarrollar la auditoría será la posibilidad que nos dará de analizar hasta qué punto la cultura informacional de la organización está alineada con sus objetivos.

\section{«La información, a pesar de ser un recurso vital para la organi- zación, no está lo suficiente- mente valorada por la estrate- gia empresarial»}

Finalmente, la auditoría nos permitirá evaluar los costes y beneficios de la gestión de información que está llevando a cabo la empresa en función de su adecuación con los parámetros descritos. Además, al final también estaremos en condiciones de determinar el valor que se le concede a la información dentro de la organización.

\section{Fases para establecer la auditoría de la información}

Aunque existen diferentes metodologías para llevarla a cabo, a nivel general destacamos las siguientes etapas:

-Planificación. Supone la definición de los objetivos, saber cuál es el punto de partida y hasta dónde se pretende llegar. Entre sus tareas se encuentran las de identificar las personas clave en la organización, la envergadura tanto física como informacional del proyecto y la de localizar los recursos humanos, financieros, técnicos, etc. En esta fase habrá que decantarse por una metodología y desarrollar un plan de acción para la implementación, un plan estratégico y de comunicación y un plan de negocio.

- Colección de datos. Prepararse para la información que se va a recibir, desarrollando bases de datos, cuestionarios, entrevistas, etc.

-Análisis de datos. En esta fase se preparan los datos y los métodos de análisis.

\section{IweTel}

Es un foro electrónico de debate, puesto en marcha por EPI - El profesional de la información, sobre información, documentación, bi-

blioteconomía y sus tecnologías.

En la actualidad cuenta con más de 4.600 suscriptores.

Para suscribirse a IweTel hay que enviar a la dirección:

listserv@listserv.rediris.es

un mensaje en cuyo cuerpo figure:

subscribe iwetel Nombre Apellido

Se puede participar en IweTel remitiendo los mensajes a:

iwetel@listserv.rediris.es

Más información en:

http://www.rediris.es/list/info/iwetel.html

—Evaluación de datos. Aquí se localizan vacíos y duplicados. Esto servirá para interpretar el flujo de información, evaluar los problemas, formular recomendaciones y desarrollar un plan de acción para el cambio.

- Una vez superada la etapa anterior, hay que comunicar recomendaciones para paliar las irregularidades detectadas y dar difusión del trabajo realizado, con la finalidad de que el entorno organizacional y corporativo tenga información sobre los resultados de un trabajo en el que ellos han participado activamente.

- Implantar las recomendaciones. Desarrollar un programa de implementación, incorporar los cambios dentro de los planes formales (marketing, negocios y estrategia), desarrollar una estrategia de post-implementación y una política de información.

- Una vez realizados los cambios hay que ser consciente de la necesidad de un seguimiento continuo, medir y valorar los cambios y planificar un ciclo de auditoría de información cíclica.

\section{Conclusiones}

La auditoría de la información dará a las organizaciones, en definitiva, la posibilidad de conocer cómo trabajan en relación a la información y, en consecuencia, respecto al conocimiento. Proporcionará una 'fotografía' del uso de la información que permitirá a su vez la identificación de las áreas de la organización que estén produciendo conocimiento y aquellas donde haya una necesidad de implantación de mecanismos para la transferencia de los mismos.

Así, una vez realizada la auditoría la organización, se encontrará en disposición de desarrollar o mejorar su estrategia de gestión de la información y será capaz de sustentar el desarrollo de una correcta estrategia de gestión del conocimiento que, además, le permitirá acceder a sus fuentes de conocimiento, explotarlo mediante unos valores, una cultura y un liderazgo trans- 
formador que lo potencie y dinamice, transferir o compartir formalmente los conocimientos a través del trabajo en equipo, utilizar la tecnología como un instrumento que facilita el aprendizaje y, por último, producir nuevo conocimiento como consecuencia de éste aprendizaje organizacional, ya sea individual o colectivo.

La auditoría de la información se presenta por tanto como punto de partida de la gestión del conocimiento o lo que es lo mismo, como medio para saber qué conoce una organización.

\section{Bibliografía}

Birkbeck University of London. Information strategy. Consultado el: 2801-03.

http://www.bbk.ac.uk/is

Bouthillier, France; Shearer, Kathleen. "Understanding knowledge management and information management: the need for an empirical perspective". En: Information research. Consultado el: 05-01-03. http://informationr.net/ir/8-1/paper141.html

Brugarolas Ros, Carmen M.; Andreu Felipe, Isabel; Cárceles Andreu, José Luis; Hernández Martín, María. “Aportaciones del 'Pakm': aspectos prácticos de la gestión del conocimiento". En: VII Jornadas españolas de documentación, Fesabid 2000. Zarautz: Servicio Editorial de la Universidad del País Vasco, 2000.

Buchanan, S.; Gibb, F. "The information audit: an integrated strategic approach". En: International journal of information management, 1998, v. 18 , n. 1 , pp. $29-47$.

Bustelo, Carlota; Amarilla, Raquel. "Gestión del conocimiento y gestión de la información". En: Boletín del Instituto Andaluz de Patrimonio Histórico, 2001, marzo, v. VIII, n. 34, pp. 226-230.

Bustelo, Carlota. "Gestión documental en las empresas: una aproximación práctica". En: VII Jornadas españolas de documentación, Fesabid 2000. Zarautz: Servicio Editorial de la Universidad del País Vasco, 2000, pp. 189-195.

Bustelo, Carlota; García-Morales, Elisa. "La consultoría en organización de la información". En: El profesional de la información, 2000, septiembre, v. 9, n. 9, pp. 4-10.

"Catalunya en la societat de la informació: realitat i reptes". En: 8es. Jornades catalanes de documentació. Barcelona: Col-legi Oficial de Bibliotecaris-Documentalistes de Catalunya (Cobdc), 2001, pp. 23-33.

Cornella, Alfons. "Aprender tendiendo puentes mentales". En: El profesional de la información, 2000, abril, v. 9, n. 4, p. 36.

Cornella, Alfons. Infonomia!com: la empresa es información. Bilbao: Ediciones Deusto, 2000. Isbn 84-234-1760-3.

Coopers \& Lybrand; Jisc's Information Strategies Steering Group. Guidelines for developing an information strategy. Consultado el: 28-01-03.

http://www.jisc.ac.uk/pub/infstrat

Cortex, Edwin M.; Kazlauskas, Edward J. "Information policy audit: a case study of an organizational analysis tool". En: Special libraries, 1996, Spring, pp. 88-97.

Davenport, Thomas H. Ecología de la información: por qué la tecnología no es suficiente para lograr el éxito en la era de la información. New York: Oxford University Press, 1999. Isbn 970-613-448-4.

Davenport, Thomas H.; Prusak, Laurence. Working knowledge: how organizations manage what they know. Boston, Massachusetts: Harvard Business School Press, 1997. Isbn 0-087584-655-6.

Dobson, Chris. Beyond the information audit: checking the health of an organization's information system. Consultado el: 22-12-02.

http://www.infotoday.com/searcher/jul02/dobson.htm
Drucker, Peter F. "The coming of the new organization”. En: Harvard business review, 1988, v. 66, n. 1, pp. 45-53.

Entorno empresarial del siglo XXI; Gestión del conocimiento. IV Jornadas internacionales del clúster del conocimiento. Donostia-San Sebastián: Clúster del conocimiento, 2001.

Henczel, Susan. The information audit: a practical guide. München: Saur, 2001. Isbn 3-598-24367-7.

Henczel, Susan. "The information audit as a first step towards effective knowledge management: an opportunity for the special librarian”. En: Inspel, 2000, v. 34, n. 3/4, pp. 210-226.

Huang, Kuan-Tsae; Lee, Yang W.; Wang, Richard Y. Calidad de la información y gestión del conocimiento. Madrid: Aenor, 2000. Isbn 848143-190-7.

Hylton, Ann. A KM initiative is unlike to succeed without a knowledge audit. Consultado el: 28-01-03.

http://www.annhylton.com/writings/writings-home.htm

Lindsey-King, Cathy. Information audits: a first link in the knowledge management chain. Consultado el: 15-01-03.

http://members.rogers.com/lindsey-king/info-aud.pdf

Martín Mejías, Pedro. "De la auditoría de la información a intranet: claves para la implantación de sistemas de gestión de información en las empresas". En: Fesabid 98: VI jornadas españolas de documentación. Consultado el: 28-01-03.

http://www.fesabid98.florida-uni.es/comunicaciones/p-martin.htm

McCracken, Campbell. "Illumination, not enumeration". En: The Dialog magazine. Consultado el: 17-05-02.

http://quantum.dialog.com/q2_resources/magazine/articles/illumination.pdf

Office of Information Technology. Information management audit guideline. Consultado el: 28-01-03.

http://www.oit.nsw.gov.au/pages/4.3.12-IM-Audit.htm

Orna, Elizabeth. Practical information policies. Aldershot: Grower, 1999. Isbn 0-566-07693-4.

Oxbrow, Nigel. Information audits: the route to getting value from your intranet. Consultado el: 28-01-03.

http://www.tfpl.com/about_TFPL/reports_research/information_audits_a rticle/information_audits_article.html

Pérez Rodríguez, Judit; Coutin Domínguez, Adrián. "La gestión del conocimiento: un nuevo enfoque en la gestión empresarial". En: Gestiondelconocimiento.com. Consultado el: 02-05-02.

http://www.gestiondelconocimiento.com/documentos2/acoutin/zip/gestion. $p d f$

Ramjaun, Ibrahim. "The information audit: a tool for visionary organisations”. En: Prosi magazine, 2000, August, n. 379. Consultado el: 28-01-03. http://www.prosi.net/mag2000/379aug/audit379.htm

Sánchez Montero, José Antonio. "Intranets corporativas y gestión del conocimiento: el caso de una empresa el sector de la energía". En: VII Jornadas españolas de documentación, Fesabid 2000. Zarautz: Servicio Editorial de la Universidad del País Vasco, 2000.

Soy, Cristina. "Auditar la información... ¿para qué?”. En: Boletín de la Sedic, 2002, primavera, n. 38, pp. 1-3.

Soy, Cristina. Auditoría de la informació. Barcelona: Universitat Oberta de Catalunya, 2001. [Recurso electrónico].

Soy, Cristina; Casaldàliga, Núria. "L'auditoria de la informació com a eina per millorar l'input d'informació dels usuaris en un entorn corporatiu". En: 6es Jornades catalanes de documentació: biblioteques, centres de documentació i serveis d'informació. Barcelona: Socadi, Cobdc, 1997, pp. 49-64.

Susana Serrano González, GTD, Ingeniería de Sistemas y de Software.

susana.serrano@gtd.es

Mònica Zapata Lluch, Fundación Ciutat de Viladecans. monica@fundacionviladecans.org 